\title{
The first case in Asia of 2-methyl-3-hydroxybutyryl-CoA dehydrogenase deficiency (HSD10 disease) with atypical presentation
}

\author{
Toshiyuki Fukao ${ }^{1,2}$, Kazuhisa Akiba ${ }^{3}$, Masahiro Goto ${ }^{4}$, Nobuki Kuwayama ${ }^{1}$, Mikiko Morita ${ }^{1}$, Tomohiro Hori ${ }^{1}$, \\ Yuka Aoyama $^{2}$, Rajaram Venkatesan ${ }^{5}$, Rik Wierenga ${ }^{5}$, Yohsuke Moriyama ${ }^{6}$, Takashi Hashimoto ${ }^{6}$, \\ Nobuteru Usuda $^{6}$, Kei Murayama ${ }^{7}$, Akira Ohtake ${ }^{4,8}$, Yuki Hasegawa ${ }^{9}$, Yosuke Shigematsu ${ }^{10}$ \\ and Yukihiro Hasegawa ${ }^{4}$
}

2-Methyl-3-hydroxybutyryl-CoA dehydrogenase (2M3HBD) deficiency (HSD10 disease) is a rare inborn error of metabolism, and $<30$ cases have been reported worldwide. This disorder is typically characterized by progressive neurodegenerative disease from 6 to 18 months of age. Here, we report the first patient with this disorder in Asia, with atypical clinical presentation. A 6-year-old boy, who had been well, presented with severe ketoacidosis following a 5-day history of gastroenteritis. Urinary organic acid analysis showed elevated excretion of 2-methyl-3-hydroxybutyrate and tiglylglycine. He was tentatively diagnosed with $\beta$-ketothiolase (T2) deficiency. However, repeated enzyme assays using lymphocytes showed normal T2 activity and no T2 mutation was found. Instead, a hemizygous c.460G >A (p.A154T) mutation was identified in the HSD17B10 gene. This mutation was not found in 258 alleles from Japanese subjects (controls). A normal level of the HSD17B10 protein was found by immunoblot analysis but no 2M3HBD enzyme activity was detected in enzyme assays using the patient's fibroblasts. These data confirmed that this patient was affected with HSD10 disease. He has had no neurological regression until now. His fibroblasts showed punctate and fragmented mitochondrial organization by MitoTracker staining and had relatively low respiratory chain complex IV activity to those of other complexes.

Journal of Human Genetics (2014) 59, 609-614; doi:10.1038/jhg.2014.79; published online 18 September 2014

\section{INTRODUCTION}

HSD10 disease, originally described as 2-methyl-3-hydroxybutyryl-CoA dehydrogenase $(2 \mathrm{M} 3 \mathrm{HBD})$ deficiency, ${ }^{1}$ is a rare X-linked recessive disorder caused by a mutation in the HSD17B10 gene. ${ }^{2-5}$ This gene encodes a multifunctional protein that has $17 \beta$-hydroxysteroid dehydrogenase activity as well as $2 \mathrm{M} 3 \mathrm{HBD}$ activity, ${ }^{3-5}$ and which is also an essential component of mitochondrial RNase P, being required for tRNA processing in mitochondria. ${ }^{6}$

This disorder was first identified in a patient with progressive infantile neurodegeneration whose urinary organic acid profile was suspected to be due to $\beta$-ketothiolase (mitochondrial acetoacetyl-CoA thiolase; T2) deficiency in isoleucine catabolism. ${ }^{1}$ However, the clinical presentation of that patient was different from that of typical T2 deficiency, which is characterized by intermittent ketoacidosis and no clinical symptoms between crises, and typically normal development. ${ }^{7,8}$ Fewer than
30 patients have been reported to date. ${ }^{1,2,5,9-21}$ Typically, HSD10 disease is characterized by a progressive neurodegenerative course from 6 to 18 months of age, in conjunction with retinopathy and cardiomyopathy, leading to death at the age of $2-4$ years or later. ${ }^{5}$ However, clinical heterogeneity is noted in this disorder. ${ }^{5}$ An atypical milder presentation was reported in three families. ${ }^{13,14,17}$

Here, we describe a 6-year-old Japanese boy with the HSD10 disease, who had no neurodegeneration and developed severe ketoacidosis at the age of 6 years. This is believed to be the first report of HSD10 disease in Asia.

\section{MATERIALS AND METHODS}

Case presentation

We report the case of a boy who had been well and achieved normal development until 6 years of age when he presented with severe ketoacidosis following

${ }^{1}$ Department of Pediatrics, Graduate School of Medicine, Gifu University, Gifu, Japan; ${ }^{2}$ Medical Information Sciences Division, United Graduate School of Drug Discovery and Medical Information Sciences, Gifu University, Gifu, Japan; ${ }^{3}$ Department of General Pediatrics, Tokyo Metropolitan Children's Medical Center, Tokyo, Japan; ${ }^{4}$ Department of Endocrinology and Metabolism, Tokyo Metropolitan Children's Medical Center, Tokyo, Japan; ${ }^{5}$ Faculty of Biochemistry and Molecular Medicine and Biocenter Oulu, University of Oulu, Oulu, Finland; ' ${ }^{6}$ epartment of Anatomy and Cell Biology, Fujita Health University School of Medicine, Toyoake, Japan; ${ }^{7}$ Department of Metabolism, Chiba Children's Hospital, Chiba, Japan; ${ }^{8}$ Department of Pediatrics, Saitama Medical University, Moroyama, Japan; ${ }^{9}$ Department of Pediatrics, Shimane University School of Medicine, Izumo, Japan and ${ }^{10}$ Department of Health Science, Faculty of Medical Sciences, University of Fukui, Eiheiji-cho, Japan

Correspondence: Professor T Fukao, Department of Pediatrics, Graduate School of Medicine, Medical Information Sciences Division, United Graduate School of Drug Discovery and Medical Information Sciences, Gifu University, Gifu 501-1194, Japan.

E-mail: toshi-gif@umin.net

Received 21 January 2014; revised 24 April 2014; accepted 20 August 2014; published online 18 September 2014 
a 5-day period of appetite loss and vomiting due to gastroenteritis. Physical examination at admission showed a height of $108 \mathrm{~cm}$, body weight of $18.3 \mathrm{~kg}$ ( $2 \mathrm{~kg}$ loss), heart rate of 128 per min and respiratory rate of 32 per min. Unconsciousness was not noted. Laboratory testing showed blood gas $\mathrm{pH} 7.01$, $\mathrm{pCO}_{2} 9.2 \mathrm{~mm} \mathrm{Hg}, \mathrm{HCO}_{3}^{-} 2.8 \mathrm{mEq}^{-1}$, blood glucose $5.9 \mathrm{mmoll}^{-1}$, white blood cell count $16180 \mathrm{\mu l}^{-1}$, hemoglobin $14.3 \mathrm{~g} \mathrm{dl}^{-1}$, blood urea nitrogen 14.5 $\mathrm{mg} \mathrm{dl}^{-1}$, aspartate aminotransferase $29 \mathrm{IUl}^{-1}$, alanine aminotransferase 17 $\mathrm{IUl}^{-1}$, lactate dehydrogenase $238 \mathrm{IUl}^{-1}$, ammonia $65 \mathrm{~g} \mathrm{dl}^{-1}$ and lactate $2.4 \mathrm{mmoll}^{-1}$.

After bolus infusion of $20 \mathrm{ml} \mathrm{kg}^{-1} 5 \%$ glucose and electrolytes, blood total ketone body level was $14 \mathrm{mmoll}^{-1}$ and free fatty acid was $0.97 \mathrm{mmoll}^{-1}$. He responded to intravenous fluid infusion (including $5 \%$ glucose), and blood gas showed $\mathrm{pH} 7.48$ and $\mathrm{HCO}_{3}{ }^{-} 23.7 \mathrm{mmoll}^{-1}$ on day 2 of hospitalization. He became well and started oral food intake on that day. He was discharged from the hospital on day 7 of hospitalization. Semiquantitative urinary organic acid analysis in the acute phase showed elevated excretion of 2-methyl-3hydroxybutyrate and tiglylglycine, as well as ketones. He was tentatively diagnosed with T2 deficiency. One month later, he developed an episode of abdominal pain and lethargy in which hypoglycemia $\left(1.4 \mathrm{mmoll}^{-1}\right)$ and mild metabolic acidosis (blood $\mathrm{pH} 7.29, \mathrm{pCO}_{2} 36.4 \mathrm{~mm} \mathrm{Hg}, \mathrm{HCO}_{3}^{-} 17.5 \mathrm{mmoll}^{-1}$ and lactate $5.5 \mathrm{mmoll}^{-1}$ ) were noted. He responded quickly to intravenous infusion of electrolytes and glucose. Urinary organic acid analysis at the acute phase of this episode showed elevated concentrations of 2-methyl-3hydroxybutyrate but not of tiglylglycine and 2-methylacetoacetate (Table 1). Blood acylcarnitine analysis using tandem mass spectrometry showed elevated C5:1 carnitine but not C5-OH carnitine (Table 1). After this episode, he did not experience another metabolic event until now (6.5 years of age).

His mother claimed that his gross motor development was slow and he could walk alone after the age of 1 year and 6 months. He also had some clumsiness with fine motor skills. His growth was normal. His height and weight were $111.5 \mathrm{~cm}$ ( $-1.2 \mathrm{s.d}$.) and $22.2 \mathrm{~kg}(0$ s.d.), respectively. His neurological development was slightly below normal with a verbal IQ of 112, performance IQ of 64 and a full scale IQ of 88 (Wechsler Intelligence Scale for Children). Cerebral magnetic resonance imaging and magnetic resonance spectroscopy yielded normal findings at the age of 6.5 years. No abnormal findings were identified in echocardiography and ophthalmological examinations at the age of 7 years.

\section{Enzyme assay and immunoblot analysis}

Peripheral blood mononuclear cells were isolated from heparinized blood by gradient centrifugation in Ficoll-Paque medium (GE Healthcare, Uppsala, Sweden). The fibroblasts were cultured in Eagle's minimum essential medium containing $10 \%$ fetal calf serum. Acetoacetyl-CoA thiolase and succinyl-CoA:3- ketoacid CoA transferase were assayed in lymphocytes and fibroblasts, as described previously. ${ }^{22} 2 \mathrm{M} 3 \mathrm{HBD}$ activity in fibroblasts was measured as described previously. ${ }^{1}$ Immunoblot analysis for $2 \mathrm{M} 3 \mathrm{HBD}$ was done using anti-rat $2 \mathrm{M} 3 \mathrm{HBD}$ antibody, which was originally made by us $(\mathrm{TH})$ and antihuman glyceraldehyde 3-phosphate dehydrogenase antibody (sc-25778; Santa Cruz Biotechnology, Santa Cruz, CA, USA) as a reference. We used fibroblasts from an HSD10-deficient patient, ${ }^{16}$ as a positive disease control.

\section{Mutation analysis}

This study was approved by the Ethical Committee of the Graduate School of Medicine, Gifu University, Gifu, Japan. Genomic DNA was purified from the fibroblasts with Sepa Gene kits (Sanko Junyaku, Tokyo, Japan). Mutation screening was performed at the genomic level by PCR and direct sequencing, using primer sets for fragments including each exon and its intron boundaries. Primers and PCR conditions for ACAT1 gene were as previously described. ${ }^{23}$ For HSD17B10, we amplified each genomic region with the primer pairs shown in Supplementary Table S1.

\section{Screening of A154T mutation in the Japanese population}

The presence of A145T mutations was screened using TaqMan triplet genotyping in 92 Japanese men and 83 women, according to the manufacturer's protocol (Life Technologies, Carlsbad, CA, USA).

\section{Mitochondrial morphology}

Fibroblasts from HSD10 patients and control fibroblasts were cultured in Dulbecco's modified Eagle's medium (Life Technologies) supplemented with $10 \%$ fetal calf serum at $37^{\circ} \mathrm{C}$ and $5 \% \mathrm{CO}_{2}$. The mitochondria in living fibroblasts were stained with $100 \mathrm{~nm}$ MitoTracker Red CMRXRos (Life Technologies) for $30 \mathrm{~min}$ at $37^{\circ} \mathrm{C}$. Fluorescent images were captured and analyzed with an LSM710 laser scanning confocal microscope equipped with an incubation system (Carl Zeiss, Oberkochen, Germany).

\section{Respiratory chain enzyme analysis}

An in vitro respiratory chain enzyme activity assay ${ }^{24}$ and blue native polyacrylamide gel electrophoresis ${ }^{25,26}$ were used to quantify the activity and amount of respiratory chain enzyme complexes. The diagnostic criteria of Bernier et al. ${ }^{26,27}$ were used to judge the activity.

\section{Structural analysis of the A154 mutation}

The crystal structure of human HSD17B10 complexed with NAD ${ }^{+}$(PDB ID: $2 \mathrm{O} 23$, deposited in the RCSB protein databank; www.rcsb.org $)^{28}$ was used for

Table 1 Urinary organic acid and serum acylcarnitine analyzes

\begin{tabular}{|c|c|c|c|c|c|}
\hline & \multirow[b]{2}{*}{ Mean (s.d.) } & \multicolumn{2}{|c|}{ This patient } & \multirow{2}{*}{$\frac{\text { T2D (severe) }}{\text { Asymptomatic }}$} & \multirow{2}{*}{$\frac{T 2 D \text { (mild) }}{\text { Symptomatic }}$} \\
\hline & & Hypoglycemic & Asymptomatic & & \\
\hline \multicolumn{6}{|l|}{ Urinary organic acids } \\
\hline Lactic acid & $37.9 \pm 28.1$ & 7755.8 & 7.3 & 5.1 & 195.0 \\
\hline 3-OH butyric acid & $27.8 \pm 21.5$ & 17116.1 & 3.0 & 5.4 & 6295.0 \\
\hline Acetoacetic acid & $0.2 \pm 0.4$ & 72.5 & 0.7 & $1.0^{\mathrm{a}}$ & $16.7^{\mathrm{a}}$ \\
\hline 2-Me-3-OH butyric acid & $4.4 \pm 4.0$ & 296.2 & 132.6 & 130.4 & 121.6 \\
\hline 2-Methylacetoacetic acids & $0 \pm 0$ & 0.0 & 0.7 & $69.4^{\mathrm{a}}$ & $2.8^{\mathrm{a}}$ \\
\hline Tiglylglycine & $2.2 \pm 4.3$ & 0.1 & 298.9 & 212.4 & 3.7 \\
\hline \multicolumn{6}{|l|}{ Serum acylcarnitines } \\
\hline $\mathrm{CO}$ & $31.3 \pm 8.4$ & 13.4 & & 67.4 & 79.2 \\
\hline $\mathrm{C} 2$ & $6.2 \pm 2.1$ & 16.2 & & $7.7^{a}$ & $2.1^{\mathrm{a}}$ \\
\hline C5:1 & $0.012 \pm 0.005$ & 0.63 & & 0.72 & 0.079 \\
\hline $\mathrm{C} 5 \mathrm{OH}$ & $0.06 \pm 0.03$ & 0.11 & & 0.34 & 0.06 \\
\hline
\end{tabular}

T2D (severe) was GK01, and T2D (mild) was GK77.

Amounts of urinary organic acids are expressed as mmol per mol $\mathrm{Cr}$.

Amounts of serum acylcarnitine are expressed as nnom ml-1.

avalues may be low because of degradation due to long storage at $-30^{\circ} \mathrm{C}$ 
structural analysis. The program COOT was used to analyze the structure and PyMOL Molecular Graphics System, version 1.4.1 (Schrödinger, LLC; www. pymol.org/citing), was used to make the figures.

\section{RESULTS}

Exclusion of the diagnosis of T2 deficiency

We first made a tentative diagnosis of T2 deficiency, based on the severe ketoacidotic event with elevated 2-methyl-3-hydroxybutyrate and tiglylglycine in urinary organic acid analysis. However, repeated enzyme assays showed normal T2 activity (Supplementary Table S2). Furthermore, no T2 mutation was identified by genomic PCR followed by direct sequencing.

\section{Mutation analysis of HSD17B10 gene}

Urinary organic acid analysis showed blockade at the T2 or $2 \mathrm{M} 3 \mathrm{HBD}$ level in the isoleucine catabolic pathway. Therefore, we investigated the possibility of an HSD17B10 gene mutation, although the clinical course of this patient was different from that of typical HSD10 patients. A hemizygous c.460G $>$ A (p.A154T) mutation was identified in HSD17B10 gene (Figure 1). His mother was a heterozygous carrier of this mutation. His maternal uncle did not have this mutation. Samples from maternal grandparents were not available for the study. TaqMan analysis showed that this mutation was not found in 258 alleles from Japanese subjects (controls).

\section{Enzyme assay and immunoblot analysis for $2 \mathrm{M} 3 \mathrm{HBD}$}

We used a fibroblast cell line from a Dutch patient whose mutation was c.364C $>\mathrm{G}$ (p.L122V) as a positive disease control. He was classified with the infantile form of HSD10 disease because he had shown motor delay and spastic diplegia since infancy. ${ }^{16}$ The patient was able to walk but had psychomotor retardation with spasticity and minimal language development (Bwee Tien Poll-The, personal communication), and hence his clinical manifestations were milder than for the typical infantile form of the disease.

2M3HBD activity was absent from the patient's fibroblasts, as well as HSD10-deficient fibroblasts with p.L122V mutation, ${ }^{16}$ designated as L122V fibroblasts (Table 2). However, the control samples showed $2 \mathrm{M} 3 \mathrm{HBD}$ activity, which was in accordance with reported control values for the assay. ${ }^{1}$ Immunoblot analysis showed that fibroblasts

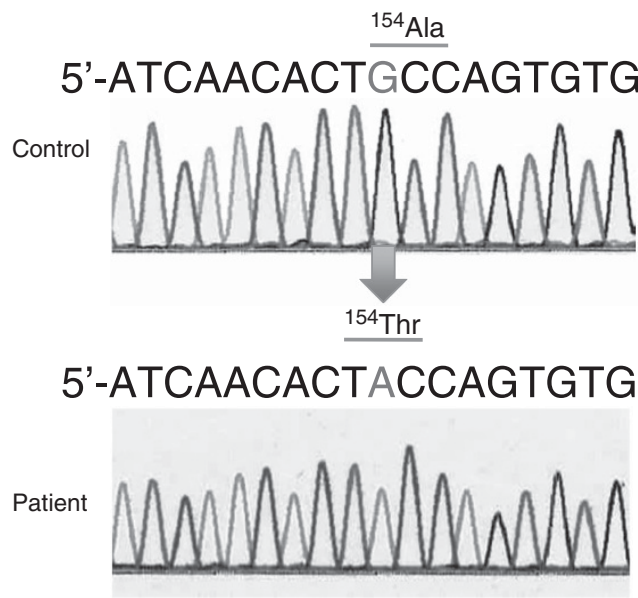

Figure 1 HSD17B10 mutation. Genomic direct sequencing of exon 5. A hemizygous c.460G $>$ A (p.A154T) substitution was identified. A full color version of this figure is available at the Journal of Human Genetics journal online. from our patient and the previous HSD10-deficient patient had an almost similar amount of HSD17B10 protein to the controls (Supplementary Figure S1).

\section{Mitochondrial staining}

MitoTracker staining revealed a filamentous network-like structure of the mitochondria in control fibroblasts (Figure 2 and Supplementary Figure S2). Fibroblasts with the p.L122V and p.A154T mutations showed punctate and fragmented mitochondrial organization. This finding is the same as that previously reported in fibroblasts with R130C and D86G mutations. ${ }^{17}$ Furthermore, mitochondria in A154T mutated cells had highly variable diameters, ranging from thin tubes to swollen bulbs.

\section{Respiratory chain enzyme assay}

Respiratory chain enzyme assay of the patient's fibroblasts showed normal activity of complexes I, II and III (98-159\% relative to citrate synthase) (Supplementary Table S3). Complex IV activity was also within the normal range but significantly lower than that of other complexes $(51.6 \%$ relative to citrate synthase and $44.6 \%$ relative to complex II). In blue native polyacrylamide gel electrophoresis, the band corresponding to assembled complex IV was slightly decreased too (Supplementary Figure S3). These tendencies were also detected in fibroblasts with L122V mutation.

Mutation site in the tertiary structure of human HSD17B10 HSD17B10 is a tetramer consisting of four identical subunits, each having the fold of short-chain dehydrogenase/reductase superfamily. Inspection of the human HSD17B10 structure (PDB ID: 2O23) revealed that residue Ala154 is close to the active site (Figure 3a). Ala154 is completely buried and the $\mathrm{C} \beta$ atom of Ala154 faces a hydrophobic (apolar) pocket created by residues such as Ile175, Val176 and Cy of Thr195. The residue next to Ala154, Ser155, is one of the catalytic residues, and part of the catalytic triad formed by Ser155, Tyr168 and Lys172. The mutation of Ala154 to Thr154, that is, from a small, hydrophobic side chain to a larger, polar side chain results in steric clashes with residues Ile175, Val176 and Thr195 in the current conformation (Figure $3 \mathrm{~b}$ ). To avoid these steric clashes, main and side chain conformational changes are expected in the region around Ile175 and Ala/Thr154. The changes around Ile175 may also affect the catalytically competent conformation of the active site residue Lys172. In addition, the changes around Ala/Thr154 are expected to cause structural changes of the catalytic residue Ser155, which has to interact with the substrate for the reaction to occur. Therefore, all these rearrangements resulting in the non-optimal conformations of Ser155 and Lys172 may severely affect the catalytic capability of this enzyme. The substrate binding may not be affected as much because the catalytic triad is only at the beginning of the much larger substrate binding pocket ${ }^{28}$ extending outward. Therefore, catalysis of both the steroid substrates such as allopregnanolone ${ }^{21}$

Table 2 2M3HBD assay using fibroblasts

\begin{tabular}{lcc}
\hline & $2 M 3 H B D$ & AcAcCOA thiolase \\
\hline Control fibroblasts 1 & $0.75 \pm 0.40$ & 15.6 \\
Control fibroblasts 2 & $0.90 \pm 0.58$ & 28.1 \\
L122V fibroblasts & $0.19 \pm 0.08$ & 28.0 \\
Patient's fibroblasts & $0.04 \pm 0.11$ & 34.0 \\
\hline
\end{tabular}

Acetoacetyl-CoA $(A \subset A c C \circ A)$ thiolase activity was measured in the presence of potassium ion at $37^{\circ} \mathrm{C}$. 

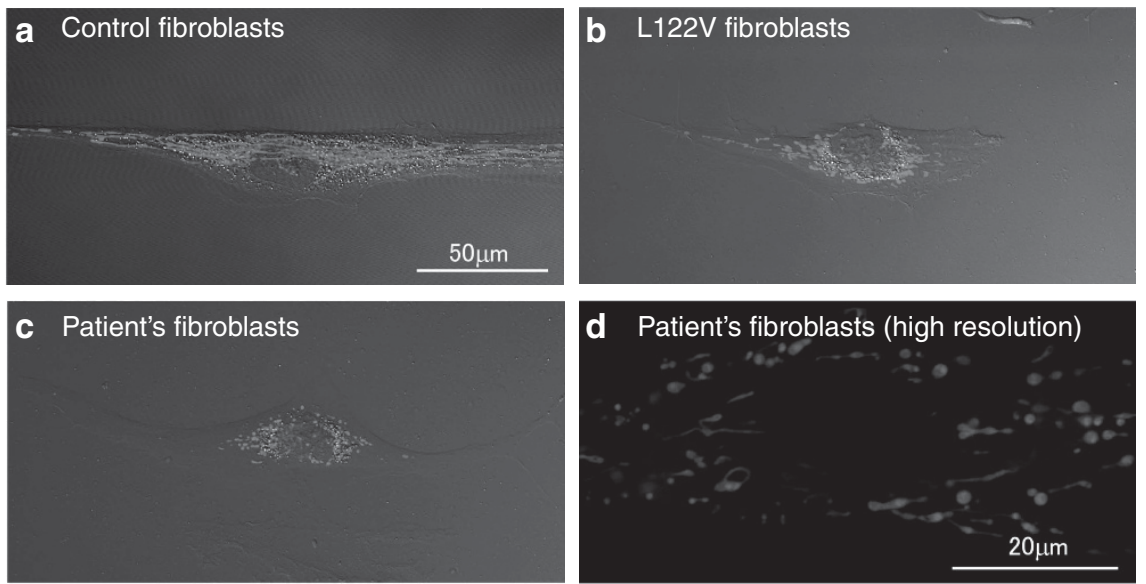

Figure 2 Mitochondrial morphology. (a-c) Merged images from differential interference contrast (DIC) and MitoTracker Red. (a) Control fibroblast. (b) Fibroblast with the p.L122V mutation. (c) Fibroblast with the p.A154T mutation. (d) Fluorescent image of MitoTracker Red from the p.A154T mutated cell. Bars: a-c, $50 \mu \mathrm{m} ; \mathbf{d}, 20 \mu \mathrm{m}$. A full color version of this figure is available at the Journal of Human Genetics journal online.

a

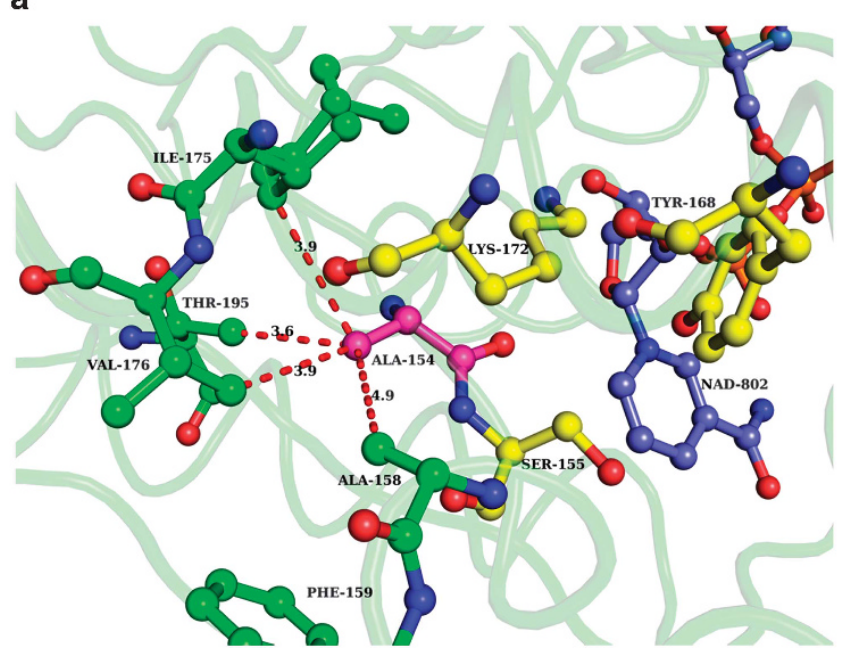

b

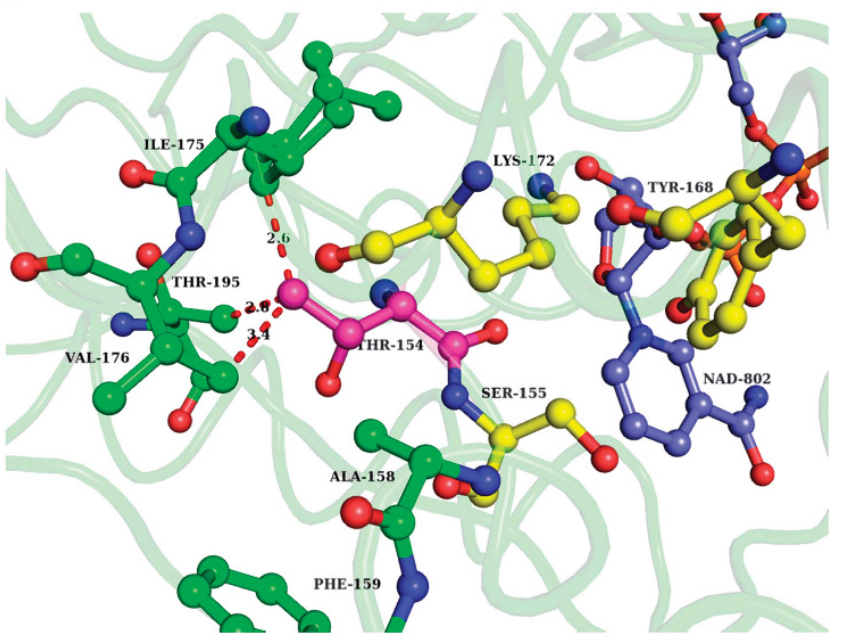

Figure 3 Structural analysis. (a) Environment of residue Ala154 as seen in PDB ID 2023. Oxygen atoms are shown in red, nitrogen in blue and carbon is color coded as follows: Ala154 in magenta, the catalytic triad comprising residues Ser155, Tyr168 and Lys172 in yellow, and NAD in blue. Ala158, Phe159, Ile175, Val176 and Thr195 (C $\gamma)$ are some of the residues pointing toward the side chain of Ala154, creating a hydrophobic pocket. These are highlighted in green. Ile175 has a double conformation. The relevant distances are shown with red dashes. (b) Possible steric clashes in HSD10 disease due to mutation of Ala154 into Thr154. Thr154 is shown in magenta. Ala154 was mutated to Thr154 using PDB-entry 2023 by the program COOT. The expected steric clashes of the Thr154 side chain with Ile175, Val176 and Thr195 are highlighted by red dashes.

and fatty acyl-CoA substrates such as 2-methyl-3-hydoxybutyryl-CoA are predicted to be equally affected.

\section{DISCUSSION}

This is believed to be the first report of HSD10 disease in Asia. Since the discovery of the first patient in $2000,{ }^{1}$ fewer than 30 patients have been described. ${ }^{1,2,5,9-21}$ Typically, this disorder is suspected when patients with neurological degeneration or psychomotor retardation show similar urinary organic acid or blood acylcarnitine profiles with T2 deficiency. However, our patient experienced a severe ketoacidotic episode with blood $\mathrm{pH} 7.01$ and blood total ketone level of $14 \mathrm{~mm}$ after a 5-day history of gastroenteritis. This clinical picture is similar to
T2 deficiency, although the onset of the first severe ketoacidotic episode at the age of 6 years is late compared with that in typical T2deficient patients who develop such crises around the age of 6 months to 2 years. ${ }^{7,8}$ The first patient described by Zschocke et al. ${ }^{1}$ had metabolic decompensation with ketonuria on day 2 of life. Disturbance in isoleucine catabolism may be attributed to such reversible metabolic decompensation in HSD10 disease, and appears to be independent from pathophysiology of neurodegeneration in HSD10 disease.

In the patients with HSD10 disease described thus far, broad clinical heterogeneity has been found. ${ }^{5,30}$ The classical presentation that is observed in most patients, which was called the infantile form by 
Zschocke ${ }^{5}$ is characterized by a period of more or less normal development in the first 6-18 months of life. This is followed by a progressive neurodegenerative disease course in conjunction with progressive cardiomyopathy, leading to death at the age of 2-4 years or older. Patients with a common mutation c.388C $>$ T (p.R130C) present with the infantile form. Some patients with other mutations have more severe neonatal forms. Atypical presentation was reported in three families. (1) Only one patient with c.745G > C (p.E249Q) mutation developed normally in the first 5 years of life and then showed neurological deterioration. ${ }^{14}$ This was classified as the juvenile form by Zschocke. ${ }^{5}$ (2) The proband of a family with c.495A > C (p. $\mathrm{Q} 165 \mathrm{H})$ mutation showed growth retardation, feeding difficulty and microcephaly but his neurological status remained normal at up to age 5 years. Moreover, his male cousin with the same mutation achieved normal neurodevelopment until his current age of 8 years, with a height and weight in the 25 th percentile. ${ }^{17}$ (3) Four boys in a large family showed X-linked intellectual disability, choreoathetosis and abnormal behavior with a normal urinary organic acid profile, and they had an apparent synonymous mutation that affected splicing efficiency in the HSD17B10 gene. ${ }^{13}$ Our patient with a novel c.460G > A (p.A154T) mutation showed no neurological degeneration, at least until age 6.5 years, and normal growth. Hence, our patient had a milder phenotype than in patients with juvenile HSD10 disease.

There is evidence that the neurological degeneration observed in HSD10 disease is not caused by a deficiency in the isoleucine metabolism-related 2M3HBD activities of HSD17B10. ${ }^{17,21}$ Instead, defects in neuroactive steroid metabolism ${ }^{21}$ and/or the non-enzymatic function of the protein required for mitochondrial integrity and cell survival $^{17}$ may be responsible for the neurological manifestations. The HSD17B10 protein is one of three component proteins of mitochondrial RNase $\mathrm{P}$, which is essential for mitochondrial translation. ${ }^{6}$ Reduced function as a component of RNase P may contribute to clinical severity. The p.R130C mutation common for infantile form reduced not only its mutant HSD10 level but also that of another RNase P component, MRPP-1, suggesting that HSD10 is important for the maintenance of the MRPP1-HSD10 subcomplex of RNase P. ${ }^{31}$ Analysis of the consequences of the A154T mutation on the tertiary structure suggests that A154T mutation affects enzyme activity of both 2-methyl-3-hydroxybutyryl-CoA and neurosteroids. The enzymological characterization of the expressed HSD17B10 A154T variant is required to confirm this observation. Mitochondrial morphological changes using MitoTracker staining have been reported, ${ }^{17}$ and we also observed punctate and fragmented mitochondrial organization in our patient. Mitochondrial respiratory chain complex IV activity was decreased in both fibroblasts with A154T and those with L122V, although the decreased level did not fulfill the minor diagnostic criteria of Bernier et al. ${ }^{27}$ Mitochondrial respiratory chain enzyme assay was reported to be normal in fibroblasts with V65A mutation. Further investigation in other fibroblasts with HSD10 disease is necessary to confirm that reduced complex IV activity is one of the characteristics in HSD10 disease.

We have described a patient with mild phenotype HSD10 disease with a novel A154T mutation, who is believed to be the first patient with HSD10 disease in Asia. Accumulation of more data on phenotype-genotype correlation of HSD10 disease is important to understand the molecular basis of the disease.

\section{CONFLICT OF INTEREST}

The authors declare no conflict of interest.

\section{ACKNOWLEDGEMENTS}

We sincerely thank Dr Jos PN Ruiter, Professor Ronald JA Wanders and Professor Bwee Tien Poll-The for providing the fibroblast cell line from an HSD10-deficient patient as a positive control and giving a protocol for the 2M3HBD enzyme assay.

1 Zschocke, J., Ruiter, J. P., Brand, J., Lindner, M., Hoffmann, G. F., Wanders, R. J. et al. Progressive infantile neurodegeneration caused by 2-methyl-3-hydroxybutyryl-CoA dehydrogenase deficiency: a novel inborn error of branched-chain fatty acid and isoleucine metabolism. Pediatr. Res. 48, 852-855 (2000).

2 Ofman, R., Ruiter, J. P., Feenstra, M., Duran, M., Poll-The, B. T., Zschocke, J. et al. 2-Methyl-3-hydroxybutyryl-CoA dehydrogenase deficiency is caused by mutations in the HADH2 gene. Am. J. Hum. Genet. 72, 1300-1307 (2003).

3 Yang, S. Y., He, X. Y. \& Miller, D. HSD17B10: a gene involved in cognitive function through metabolism of isoleucine and neuroactive steroids. Mol. Genet. Metab. 92, 36-42 (2007).

4 Yang, S. Y., He, X. Y. \& Schulz, H. Multiple functions of type 10 17beta-hydroxysteroid dehydrogenase. Trends Endocrinol. Metab. 16, 167-175 (2005).

5 Zschocke, J. HSD10 disease: clinical consequences of mutations in the HSD17B10 gene. J. Inherited Metab. Dis 35, 81-89 (2012).

6 Holzmann, J., Frank, P., Loffler, E., Bennett, K. L., Gerner, C. \& Rossmanith, W. RNase $P$ without RNA: identification and functional reconstitution of the human mitochondrial tRNA processing enzyme. Cell 135, 462-474 (2008).

7 Fukao, T., Scriver, C. R. \& Kondo, N. The clinical phenotype and outcome of mitochondrial acetoacetyl-CoA thiolase deficiency (beta-ketothiolase or T2 deficiency) in 26 enzymatically proved and mutation-defined patients. Mol. Genet. Metab. 72, 109-114 (2001).

8 Mitchell, G. A. \& Fukao, T. in The Metabolic \& Molecular Basis of Inherited Disease Vol. 2, Ch. 102 (eds Scriver, C. R., Beaudet, A. L., Sly, W. S. \& Valle D.) 2327-2356 (McGraw-Hill, New York, 2001).

9 Cazorla, M. R., Verdu, A., Perez-Cerda, C. \& Ribes, A. Neuroimage findings in 2-methyl3-hydroxybutyryl-CoA dehydrogenase deficiency. Pediatr. Neurol. 36, 264-267 (2007).

10 Ensenauer, R., Niederhoff, H., Ruiter, J. P., Wanders, R. J., Schwab, K. O., Brandis, M. et al. Clinical variability in 3-hydroxy-2-methylbutyryl-CoA dehydrogenase deficiency. Ann. Neurol. 51, 656-659 (2002).

11 Garcia-Villoria, J., Gort, L., Madrigal, I., Fons, C., Fernandez, C., Navarro-Sastre, A. et al. X-inactivation of HSD17B10 revealed by cDNA analysis in two female patients with 17beta-hydroxysteroid dehydrogenase 10 deficiency. Eur. J. Hum. Genet 18, 1353-1355 (2010).

12 Garcia-Villoria, J., Navarro-Sastre, A., Fons, C., Perez-Cerda, C., Baldellou, A., Fuentes-Castello, M. A. et al. Study of patients and carriers with 2-methyl-3hydroxybutyryl-CoA dehydrogenase (MHBD) deficiency: difficulties in the diagnosis. Clin. Biochem. 42, 27-33 (2009).

13 Lenski, C., Kooy, R. F., Reyniers, E., Loessner, D., Wanders, R. J., Winnepenninckx, B. et al. The reduced expression of the $\mathrm{HADH} 2$ protein causes $\mathrm{X}$-linked mental retardation, choreoathetosis, and abnormal behavior. Am. J. Hum. Genet. 80, 372-377 (2007).

14 Olpin, S. E., Pollitt, R. J., McMenamin, J., Manning, N. J., Besley, G., Ruiter, J. P. et al. 2-Methyl-3-hydroxybutyryl-CoA dehydrogenase deficiency in a 23-year-old man. J. Inherited Metab. Dis 25, 477-482 (2002).

15 Perez-Cerda, C., Garcia-Villoria, J., Ofman, R., Sala, P. R., Merinero, B., Ramos, J. et al. 2-Methyl-3-hydroxybutyryl-CoA dehydrogenase (MHBD) deficiency: an X-linked inborn error of isoleucine metabolism that may mimic a mitochondrial disease. Pediatr. Res. 58, 488-491 (2005)

16 Poll-The, B. T., Wanders, R. J., Ruiter, J. P., Ofman, R., Majoie, C. B., Barth, P. G. et al. Spastic diplegia and periventricular white matter abnormalities in 2-methyl-3-hydroxybutyryl-CoA dehydrogenase deficiency, a defect of isoleucine metabolism: differential diagnosis with hypoxic-ischemic brain diseases. Mol. Genet. Metab. 81, 295-299 (2004).

17 Rauschenberger, K., Scholer, K., Sass, J. O., Sauer, S., Djuric, Z., Rumig, C. et al. A non-enzymatic function of 17 beta-hydroxysteroid dehydrogenase type 10 is required for mitochondrial integrity and cell survival. EMBO Mol. Med 2, 51-62 (2010).

18 Sass, J. O., Forstner, R. \& Sperl, W. 2-Methyl-3-hydroxybutyryl-CoA dehydrogenase deficiency: impaired catabolism of isoleucine presenting as neurodegenerative disease. Brain Dev 26, 12-14 (2004).

19 Seaver, L. H., He, X. Y., Abe, K., Cowan, T., Enns, G. M., Sweetman, L. et al. A novel mutation in the HSD17B10 gene of a 10-year-old boy with refractory epilepsy, choreoathetosis and learning disability. PLOS ONE 6, e27348 (2011).

20 Sutton, V. R., O'Brien, W. E., Clark, G. D., Kim, J. \& Wanders, R. J. 3-Hydroxy-2methylbutyryl-CoA dehydrogenase deficiency. J. Inherited Metab. Dis 26, 69-71 (2003).

21 Yang, S. Y., He, X. Y., Olpin, S. E., Sutton, V. R., McMenamin, J., Philipp, M. et al. Mental retardation linked to mutations in the HSD17B10 gene interfering with neurosteroid and isoleucine metabolism. Proc. Natl Acad. Sci. USA 106, 14820-14824 (2009).

22 Fukao, T., Song, X. Q., Mitchell, G. A., Yamaguchi, S., Sukegawa, K., Orii, T. et al. Enzymes of ketone body utilization in human tissues: protein and messenger RNA levels of succinyl-coenzyme A ( $\mathrm{COA}): 3$-ketoacid $\mathrm{CoA}$ transferase and mitochondrial and cytosolic acetoacetyl-CoA thiolases. Pediatr. Res. 42, 498-502 (1997).

23 Fukao, T., Nakamura, H., Song, X. Q., Nakamura, K., Orii, K. E., Kohno, Y. et al. Charac terization of N93S, I312T, and A333P missense mutations in two Japanese families with mitochondrial acetoacetyl-CoA thiolase deficiency. Hum. Mutat. 12, 245-254 (1998). 
24 Kirby, D. M., Crawford, M., Cleary, M. A., Dahl, H. H., Dennett, X. \& Thorburn, D. R. Respiratory chain complex I deficiency: an underdiagnosed energy generation disorder. Neurology 52, 1255-1264 (1999).

25 Schagger, H. \& von Jagow, G. Blue native electrophoresis for isolation of membrane protein complexes in enzymatically active form. Anal. Biochem. 199, 223-231 (1991)

26 Kirby, D. M., Salemi, R., Sugiana, C., Ohtake, A., Parry, L., Bell, K. M. et al. NDUFS6 mutations are a novel cause of lethal neonatal mitochondrial complex I deficiency. J. Clin. Invest. 114, 837-845 (2004)

27 Bernier, F. P., Boneh, A., Dennett, X., Chow, C. W., Cleary, M. A. \& Thorburn, D. R. Diagnostic criteria for respiratory chain disorders in adults and children. Neurology 59, 1406-1411 (2002).
28 Benach, J., Filling, C., Oppermann, U. C., Roversi, P., Bricogne, G., Berndt, K. D. et al Structure of bacterial 3beta/17beta-hydroxysteroid dehydrogenase at 1.2 A resolution: a model for multiple steroid recognition. Biochemistry. 41, 14659-14668 (2002).

29 Persson, B., Kallberg, Y., Bray, J. E., Bruford, E., Dellaporta, S. L., Favia, A. D. et al. The SDR (short-chain dehydrogenase/reductase and related enzymes) nomenclature initiative. Chemico-Biological Interactions 178, 94-98 (2009).

30 Yang, S. Y., He, X. Y. \& Miller, D. Hydroxysteroid (17beta) dehydrogenase X in human health and disease. Mol. Cell Endocrinol. 343, 1-6 (2011).

31 Deutschmann, A. J., Amberger, A., Zavadil, C., Steinbeisser, H., Mayr, J. A., Feichtinger, R. G. et al. Mutation or knock-down of 17beta-hydroxysteroid dehydrogenase type 10 cause loss of MRPP1 and impaired processing of mitochondrial heavy strand transcripts. Hum. Mol. Genet. 23, 3618-3628 (2014).

Supplementary Information accompanies the paper on Journal of Human Genetics website (http://www.nature.com/jhg) 